\title{
1 Phylogenetic evidence of headful packaging strategy in gene transfer
}

\section{2 agents}

4 Emma Esterman ${ }^{1}$, Yuri I. Wolf ${ }^{2}$, Roman Kogay ${ }^{1}$, Eugene V. Koonin ${ }^{2}$, and Olga Zhaxybayeva ${ }^{1,3^{*}}$

$6{ }^{1}$ Department of Biological Sciences, Dartmouth College, Hanover, NH 03755, USA

$7 \quad{ }^{2}$ National Center for Biotechnology Information, National Library of Medicine, Bethesda, MD

8 20894, USA

$9{ }^{3}$ Department of Computer Science, Dartmouth College, Hanover, NH 03755, USA

11 *corresponding author: olga.zhaxybayeva@dartmouth.edu

12

14 Keywords: large terminase, terL, DNA packaging, Rhodobacter capsulatus, RcGTA,

15 alphaproteobacteria

16 


\section{Abstract}

Gene transfer agents (GTAs) are virus-like particles encoded and produced by many

20 bacteria and archaea. Unlike viruses, GTAs package fragments of the host genome instead of the

21 genes that encode the components of the GTA itself. As a result of this non-specific DNA

22 packaging, GTAs can transfer genes within bacterial and archaeal communities. GTAs clearly

23 evolved from viruses and are thought to have been maintained in prokaryotic genomes due to the

24 advantages associated with their DNA transfer capacity. The most-studied GTA is produced by

25 the alphaproteobacterium Rhodobacter capsulatus (RcGTA), which packages random portions of

26 the host genome at a lower DNA density than usually observed in tailed bacterial viruses. How

27 the DNA packaging properties of RcGTA evolved from those of the ancestral virus remains

28 unknown. To address this question, we reconstructed the evolutionary history of the large

29 subunit of the terminase (TerL), a highly conserved enzyme used by viruses and GTAs to

30 package DNA. We found that RcGTA-like TerLs grouped within viruses that employ the headful

31 packaging strategy. Because distinct mechanisms of viral DNA packaging correspond to

32 differences in the TerL amino acid sequence, our finding suggests that RcGTA evolved from a

33 headful packaging virus. Headful packaging is the least sequence-specific mode of DNA

34 packaging, which would facilitate the switch from packaging of the viral genome to packaging

35 random pieces of the host genome during GTA evolution. 


\section{Introduction}

Gene transfer agents (GTAs) are virus-like particles encoded and produced by certain

39 bacteria and archaea (reviewed most recently by Lang et al. (2017) and Grull et al. (2018)).

40 Unlike viruses, GTAs package fragments of the host genome instead of the genes that encode the

41 GTA itself (Hynes et al., 2012). When GTA particles infect another cell, they can transfer the

42 encapsidated genetic material to the recipient (Hynes et al., 2012; McDaniel et al., 2010; Solioz

43 et al., 1975). The genomic loci that encode GTAs resemble prophages, indicating that GTAs

44 evolved from viral ancestors. Although the function of GTAs is not firmly established, the

45 prevailing hypothesis is that GTAs are not defective prophages, but instead are agents of

46 horizontal gene transfer that are maintained in prokaryotic genomes due to the advantages

47 associated with gene exchange, particularly, in stressful conditions (Kogay et al., 2020; Lang et

48 al., 2017).

The best-studied GTA is produced by the alphaproteobacterium Rhodobacter capsulatus,

50 and will be referred to as RcGTA. Production of the RcGTA particles is triggered by

51 environmental factors (Westbye et al., 2017a), occurs in a small fraction of the population (Fogg

52 et al., 2012; Hynes et al., 2012), is regulated by host proteins (Ding et al., 2019; Fogg, 2019;

53 Westbye et al., 2017b) and involves expression of genes that are found in at least five loci in the

$54 \quad R$. capsulatus genome (Hynes et al., 2016; Lang et al., 2017). The largest of these loci is a 17-

55 gene "head-tail cluster" that encodes proteins involved in head-tail morphogenesis and DNA

56 packaging (Lang et al., 2017). There are homologs of the RcGTA head-tail cluster genes in other

57 alphaproteobacteria (Shakya et al., 2017), including several Rhodobacterales for which GTA

58 production has been observed (Fu et al., 2010; Nagao et al., 2015). Additionally, homologs of

59 head-tail cluster genes are present in numerous viruses and proviruses (Shakya et al., 2017). 
The small size of RcGTA and the low density of its packaged DNA precludes the particle

61 from accommodating all of the genes required for its production (Bárdy et al., 2020). Instead,

62 RcGTA packages seemingly random portions of the host genome (Hynes et al., 2012). In double-

63 stranded DNA viruses of the realm Duplodnaviria, genome packaging is mediated by the

64 terminase and portal proteins (Fokine and Rossmann, 2014; Rao and Feiss, 2015). Viral

65 terminases typically consist of large and small subunits (Rao and Feiss, 2008). The small subunit

66 (TerS) binds to the DNA to be packaged and then recruits the large subunit (Casjens, 2011; Rao

67 and Feiss, 2015). The large subunit (TerL), which consists of ATPase and nuclease domains, cuts

68 the concatemeric viral DNA, translocates the DNA into the viral capsid with concomitant

69 ATPase hydrolysis and, finally, cuts the DNA again to terminate packaging (Casjens, 2011; Rao

70 and Feiss, 2015). Viruses evolved different strategies for packaging DNA into their capsids, and

71 these strategies involve different classes of TerL (Casjens and Gilcrease, 2009). Some viruses

72 employ a "headful" packaging strategy in which TerL packages DNA into the capsid until it is

73 full, whereas other viral TerLs terminate packaging at specific sequences (Casjens and Gilcrease,

74 2009; Rao and Feiss, 2008).

Viral TerLs with the same packaging mechanism tend to form clades in phylogenetic

76 trees (Casjens et al., 2005). However, due to the diversity of TerL sequences, the relationships

77 among the different functional classes of TerLs are not well-resolved (Casjens and Gilcrease,

78 2009; Casjens et al., 2005; Merrill et al., 2016). Given its lack of sequence specificity, RcGTA is

79 presumed to package fragments of the host DNA via the headful strategy (Casjens et al., 2005;

80 Hynes et al., 2012). The RcGTA TerL and its alphaproteobacterial homologs formed a distinct

81 group in previous phylogenies, but they did not cluster with or within the viral TerLs that are

82 involved in headful packaging (Casjens and Gilcrease, 2009; Casjens et al., 2005). Therefore, 
83 these phylogenies did not provide evidence of a headful packaging strategy in

84 alphaproteobacterial GTAs, in part, due to limited sequence data available at the time.

In this study, we conducted a comprehensive evolutionary analysis of TerL sequences to

86 better resolve the phylogenetic relationship of alphaproteobacterial RcGTA-like TerLs and viral

87 TerLs with known packaging strategies. The results support a headful packaging strategy for

88 RcGTA and, by inference, the rest of the putative alphaproteobacterial GTAs. We also identified

89 two amino acid substitutions that are conserved in the TerLs of the putative alphaproteobacterial

90 GTAs and might be important for the DNA packaging properties of GTAs.

\section{Methods}

\section{Retrieval and Sequence-based Clustering of Large Terminase Homologs}

94 retrieved from the NCBI Conserved Domains Database (CDD) (Lu et al., 2020) (accessed on

95 December 11, 2018). Two TerL profiles for distinct families of bacterial and archaeal viruses

96 were added from Philosof et al. (2017) and Yutin et al. (2018). These 20 profiles

97 (Supplementary Table S1) were used as queries for PSI-BLAST searches (E-value threshold of

980.01 , effective database size of $2 \times 10^{7}$ sequences) (Altschul et al., 1997) against the NCBI non-

99 redundant protein database (accessed in December 2018). Only the subject sequences that were

100 taxonomically assigned to archaea, bacteria and viruses were retained.

101 Partial TerL sequences were removed by ensuring the presence of both an ATPase (N-

102 terminal) and a nuclease (C-terminal) domain using the following criteria: sequences either had

103 to align to $>=75 \%$ of a "full" TerL profile that includes both TerL domains or align to different

104 TerL profiles over their N- and C-terminal domains. A sequence was considered to align over a

105 specific domain if it met one of two conditions: 1) if the sequence matched $>=75 \%$ of an $\mathrm{N}$ - or 
106 C-terminal domain-specific profile and had at least $35 \%$ of the protein length outside of the

107 matched domain to contain the unmatched domain or 2) if a sequence aligned to just the $\mathrm{N}$ - or $\mathrm{C}$ -

108 terminal portion of a "full" TerL profile and had at least $35 \%$ of the protein length outside of the

109 matched domain to contain the unmatched domain.

110 The resulting 254,382 sequences were clustered using MMseqs2 (Steinegger and Söding,

1112017 ) with a similarity threshold of 0.75 . From each of the obtained 11,298 clusters, a

112 representative sequence of median length was selected for subsequent analyses.

114 Alignment of Representative Homologs and Filtering Out Partial Sequences

115 The representative TerL sequences were iteratively aligned and clustered using the

116 approach described by Wolf et al. (2018). Briefly, the sequences were clustered with a similarity

117 threshold of 0.5 using UCLUST (Edgar, 2010). The clustered sequences were aligned using

118 MUSCLE (Edgar, 2004) and alignment sites that contained more than 67\% gaps were

119 temporarily removed. Pairwise similarity scores between cluster alignments were calculated

120 using HHSEARCH (Söding, 2005), converted to a distance matrix and used to build a UPGMA

121 tree (Sokal and Michener, 1958). All of the branches of the UPGMA tree above a depth

122 threshold of 2.3 were used to guide progressive alignment of the clusters using HHALIGN

123 (Söding, 2005). The removed sites were reinserted back into their original sequences after the

124 profile-profile alignment. These alignment and clustering steps were repeated for 20 iterations,

125 when 11,230 of the sequences formed one alignment.

126 Of the remaining 68 sequences that failed to align, two were clearly TerLs but contained

127 inteins, which were manually removed. Five other sequences were also likely TerLs because

128 they were longer than 300 amino acids, exhibited significant similarity to a TerL profile via CDD 
129 searches (E-value $<0.001)$, and contained recognizable Walker A motif and nuclease catalytic

130 residues. The seven sequences were profile-aligned to the alignment of 11,230 sequences using

131 more relaxed criteria (similarity threshold of 0.01 and UPGMA depth threshold of 6). The

132 remaining 61 sequences did not meet these criteria and were discarded.

133 The new alignment of 11,237 sequences contained partial sequences that lacked a Walker

134 A motif. To remove these, each sequence's similarity was scored to the alignment's consensus

135 sequence using a BLOSUM62 substitution matrix and the score was compared to the score of

136 sequences with $100 \%$ identity to the consensus sequence. Sequences with a score less than $10 \%$

137 of the perfect match score were removed. Then, the alignment was used as a PSSM in a PSI-

138 BLAST search against all of the sequences within the alignment. Only the sequences that

139 matched to $>=75 \%$ of the PSSM were retained. The sections of the 11,060 sequences that passed

140 this criterion were extracted and re-aligned using the above-described iterative alignment

141 procedure with a clustering similarity threshold of 0.5 and UPGMA depth threshold of 2.3. After

14223 iterations of alignment and clustering, 11,057 of the sequences aligned. The three sequences

143 that did not align were longer than 300 amino acids, exhibited significant similarity to a TerL

144 profile via CDD searches (E-value $<0.001$ ), and contained a recognizable Walker A motif and

145 nuclease catalytic residues. Therefore, they were retained and aligned to the main alignment

146 using more relaxed parameters of a clustering similarity threshold of 0.01 and UPGMA depth

147 threshold of 6.

148

149 Alignment Trimming

150 The alignment of 11,060 sequences was trimmed to remove all columns with more than

$15150 \%$ gaps and less than $10 \%$ amino acid homogeneity. The homogeneity value of an alignment 
152 column was defined and calculated using the following procedure. For each of the $N=11,060$

153 sequences, column-based sequence weights $w_{i}\left(\sum_{i=1}^{N} w_{i}=1\right)$ were assigned according to

154 Henikoff and Henikoff (1994). The score of an alignment column against an amino acid $x$ was

155 calculated as $Q_{x}=\sum_{i=1}^{N} w_{i} S_{a_{i}, x}$, where $a_{i}$ is an amino acid in the $i$-th sequence and $S_{a_{i}, x}$ is the

156 BLOSUM62 substitution matrix score for a pair of amino acids $a_{i}$ and $x$ (Henikoff and Henikoff,

157 1993). As the consensus amino acid of the column $c$, the amino acid with the highest score $Q_{c}$,

158 i.e. $c=\underset{x}{\operatorname{argmax}} Q_{x}$, was selected. An expectation of the score of the given alignment column

159 against a randomly selected amino acid $R$ was calculated as $Q_{R}=\sum_{b} f_{b} Q_{b}$, where $f_{b}$ is the

160 vector of relative frequencies of amino acids $\left(\sum_{b} f_{b}=1, b \in\{\right.$ Ala. . Tyr $\left.\}\right)$. The homogeneity of

161 an alignment column was defined as $H=\max \left(\frac{Q_{c}-Q_{R}}{S_{c, c}-Q_{R}}, 0\right)$. The homogeneity ranges from 0 (the

162 alignment column score does not exceed the random expectation score $Q_{R}$ ) to 1 (the alignment

163 column score is equal to the maximum possible score $S_{c, c}$ ).

164

165 Reconstruction of Large Terminase Phylogeny

166 The trimmed alignment of 11,060 sequences was used to reconstruct an initial

167 phylogenetic tree in FastTree v. 2.1.4 (Price et al., 2010) using the Whelan and Goldman (WAG)

168 substitution model (Whelan and Goldman, 2001) and 20 gamma-distributed rate categories

169 (Yang, 1994). The initial tree was used as a guide tree to refine our alignment, which in turn was

170 used to reconstruct an improved tree. To this end, the sequences were divided into two sets, those

171 that formed distinct groups on the tree and the remaining ones. The sequences that formed

172 distinct groups were re-aligned using a clustering similarity threshold of 0.01 and UPGMA depth

173 threshold of 2.3, whereas the other sequences were aligned using more stringent parameters

174 (similarity threshold of 0.66 and UPGMA depth threshold of 1.3). These alignments were 
175 profile-aligned using a similarity threshold of 0.5 and UPGMA depth threshold of 2.3. Nine

176 sequences did not join the main alignment and were discarded due to low scores against the

177 consensus, calculated with a BLOSUM62 matrix as described above. The resulting alignment of

17811,051 sequences was trimmed to remove all columns with more than $50 \%$ gaps and less than

$17910 \%$ amino acid homogeneity, as defined above. The final phylogenetic tree of $11,051 \mathrm{TerL}$

180 homologs (Figure 1) was reconstructed using FastTree v. 2.1.4 (Price et al., 2010) with the

181 WAG substitution model (Whelan and Goldman, 2001) and 20 gamma-distributed rate

182 categories (Yang, 1994).

183

\section{Identification of RcGTA-like Large Terminases}

In the phylogenetic tree of TerLs (Figure 1), a group of 616 TerLs was labeled as the

187 that were identified and curated by Kogay et al. (2019). Nineteen RcGTA-like TerLs from the

188 dataset of Kogay et al. (2019) are absent in our dataset because they were not in GenBank at the

189 time of our data collection (December 2018). The 616 TerLs were classified as either "RcGTA-

190 like" or "virus-like" using a machine learning approach implemented in the GTA-Hunter

191 program (Kogay et al., 2019).

192

193 Examination of the Genomic Neighborhoods of Large Terminases

194 For the 604 TerLs within the "RcGTA-like containing" group (Figure 1) that originated

195 from bacterial and archaeal genomes, the presence of 11 other RcGTA-like genes near the terL

196 gene was examined. To this end, the RcGTA-like genes from the training set of Kogay et al.

197 (2019) were used as queries in a BlastP search against the assemblies of the bacterial and 
198 archaeal genomes (E-value $<0.001$; query and subject overlap by at least $60 \%$ of their length)

199 (Altschul et al., 1997). The detected RcGTA gene homologs were classified as "RcGTA-like" or

200 "virus-like" using GTA-Hunter (Kogay et al. 2019). The detected RcGTA gene homologs were

201 also clustered into regions using DBSCAN, with a maximum distance cutoff of 8,000 bp

202 between adjacent genes (Ester et al., 1996; Kogay et al., 2019). If a terL gene was embedded in a

203 region containing at least 6 of the 11 RcGTA-like genes, it was classified as being in a "large

204 RcGTA-like element." If a terL gene was located in a region containing 1 to 5 RcGTA-like

205 genes, it was classified as being in a "small RcGTA-like element." The classification of the

206 sequence represented in the phylogeny was assumed to be the same for the rest of the cluster

207 members although this was not directly verified.

208

209 Assignment of Packaging Strategy to Viral Large Terminases

210 A list of viruses with experimentally determined packaging mechanisms was compiled

211 from the phylogenetic tree of Casjens and Gilcrease (2009). Viruses from the phylogenetic tree

212 of Merrill et al. (2016) were also added, for most of which experimental evidence of the

213 packaging mechanism is available. The dataset of the 252,614 TerL homologs was searched for

214 these 87 viruses using TerL accession numbers provided by Merrill et al. (2016) and NCBI

215 taxonomy IDs for the viruses from Casjens and Gilcrease (2009). Of the 87 viruses, 73 were

216 present in our dataset (Supplementary Table S2). Due to the close sequence similarity, some of

217 the 73 TerLs belong to the same MMSEQ clusters, and therefore are represented by 58 TerLs on

218 our phylogeny of 11,051 TerLs (Figure 1). The 58 representative viruses for which the

219 packaging mechanism was not known were assigned the mechanism of a virus from the same 
220 cluster with a known packaging strategy, under the assumption that the similarity of their TerL

221 amino acid sequences is sufficient to imply the same packaging mechanism.

\section{Validation of the Reconstructed Phylogenetic Patterns with More Accurate Maximum}

\section{Likelihood Analyses}

To confirm that the phylogenetic relationships obtained from the FastTree program (Price

227 phylogenetic trees were reconstructed using IQ-TREE v 1.6.7 (Nguyen et al., 2015) from two 228 datasets subsampled from the 11,051 TerLs. The first dataset of 342 TerLs was constructed to

229 broadly represent the TerL diversity (Figure 1). The dataset contains the 58 representative viral

230 TerLs (described in Assignment of Packaging Strategy to Viral Large Terminases section),

23150 TerLs randomly sampled from group 1 of the "RcGTA-like containing group" (Figure 2), all

232 TerLs from group 2, 70 TerLs from group 3, 50 TerLs randomly sampled from the rest of the

233 "RcGTA-like containing group," and 100 randomly sampled TerLs from the rest of the whole

234 TerL tree (Figure 1). The second dataset of 346 TerLs was constructed to represent well the

235 TerLs from the "RcGTA-like containing group" (Figure 1 and Figure 2). The dataset contains

23612 representative viral TerLs with either P22 or Sf6-like headful packaging strategies, 70 TerLs

237 randomly sampled from group 1, all TerLs from group 2, and 250 TerLs randomly sampled from

238 group 3. For both datasets, the aligned sequences were retrieved from the trimmed alignment of

239 11,051 TerLs (see Reconstruction of Large Terminase Phylogeny section) and gap-only sites

240 were removed. The optimal evolutionary model was selected using ModelFinder

241 (Kalyaanamoorthy et al., 2017), as implemented in IQ-TREE. Support values for branches were 242 calculated using ultrafast bootstrap approximation with 1000 replicates (Hoang et al., 2018), as 
243 implemented in IQ-TREE. The tree reconstructed from the second dataset was rooted with the

244 headful P22 viral TerL that, out of the headful P22 viral TerLs in Figure 1, is the most distantly

245 related to the "RcGTA-like containing group."

To examine whether the bacterial TerLs classified as virus-like reside in prophages,

249 prophages were predicted in the corresponding nucleotide genome sequences using PHASTER

250 (Arndt et al., 2016, accessed in May 2020). The regions labeled as intact (score $>90$ ) or

251 questionable (score 70-90) prophages were retained, while the regions labeled as incomplete

252 prophages $($ score $<70)$ were discarded. One region surrounding the virus-like TerL (accession

253 WP_020474221) was predicted by PHASTER to be an intact prophage and was also classified as

254 a small RcGTA-like element by GTA-Hunter, due to the presence of one RcGTA-like gene. The

255 PHASTER prediction of this region as a prophage was considered to supersede the small

256 RcGTA-like element classification.

Detection of Conserved Sites that Differentiate RcGTA-Like and Virus-Like TerLs

The amino acid sequences of the 616 TerLs in the subtree shown on Figure 2 were re-

260 aligned using MAFFT-linsi v. 7.305 (Katoh and Standley, 2013). The alignment was scanned for

261 sites conserved in more than $80 \%$ of the TerL homologs from group 1 (virus-like) or group 3

262 (RcGTA-like) (Figure 2). Of these detected sites, only the sites with at least 70\% between-group

263 difference in the relative abundance of the most conserved amino acid were retained.

The positions of the two identified sites relative to the known TerL structural domains

265 were determined by searching CDD (Lu et al., 2020) with RcGTA TerL RefSeq record 
WP_031321187 as a query (database accessed on July 26, 2020). The conservation of the two

267 detected sites within the "RcGTA-like containing group" was visualized using a subset of the

268616 TerLs (Figure 2): 15 randomly sampled TerLs from group 1, all TerLs from group 2, 14

269 randomly sampled TerLs from group 3, and a representative TerL from the cluster that contains

270 the RcGTA TerL. The aligned TerLs were retrieved from the alignment of 616 TerLs, and the

271 gap-only alignment positions were removed. Secondary structure information was obtained via

272 HHPred using RcGTA TerL as a query (Zimmermann et al., 2018).

273 The locations of the two sites were also visualized on a 3D structure of TerL from the

274 Shigella phage Sf6 (PDB ID 4IDH) (Zhao et al., 2013) which, based on our phylogenetic

275 inference, is the TerL most closely related to RcGTA-like TerLs for which a structure is

276 available. The homologous positions of the substitutions in the Shigella phage Sf6 TerL were

277 identified by aligning it to the RcGTA TerL using HHPred (Zimmermann et al., 2018). The

278 visualization was carried out in PyMOL v 2.4 (Schrödinger, LLC, 2020).

\section{Results}

280 RcGTA-like TerLs belong within the group of TerLs of headful packaging phages

Of the 11,051 representative TerL homologs from bacteria, archaea and viruses, 616 are

282 closely related to the RcGTA TerL and form a well-supported group in the phylogenetic tree

283 (Figure 1). Of these 616 TerLs, twelve are encoded in viral genomes, whereas the remaining 604

284 are found in 601 bacterial and 3 archaeal genomes. Using a machine learning approach that relies

285 on amino acid composition, we classified the 604 bacterial and archaeal TerL homologs as either

286 "RcGTA-like” (527) or "virus-like" (77) (Supplementary Table S3). By mapping 73 TerLs

287 with experimentally determined packaging strategies onto the phylogeny, we found that RcGTA-

288 like TerLs fall, with strong support, within a group of headful packaging phages (Figure 1). 
289 Therefore, our phylogeny implies that the RcGTA-like TerLs evolved from a viral TerL that

290 employed a headful packaging strategy and thus supports the hypothesis that RcGTA-like TerLs

291 use a headful mechanism to package host DNA (Casjens et al., 2005; Hynes et al., 2012). Of the

292 TerLs from the viruses with experimentally determined packaging strategies, Enterobacteria

293 phage P22-like TerLs are the closest relatives of the RcGTA-like TerLs. This affinity contrasts

294 the previous results, from analyses of much smaller data sets, according to which T4-like (Hynes

295 et al., 2012; Lang and Beatty, 2000) or T7-like (Casjens et al., 2005) TerLs have been found to

296 be most closely related to the RcGTA-like TerLs.

Phylogenetic evidence of a single origin of GTA TerLs

Whereas the TerLs of viruses that employ headful DNA packaging specifically package

300 the viral genome into the capsid, RcGTA TerL lacks sequence specificity and packages random

301 segments of the bacterial genome (Hynes et al., 2012). To evaluate if non-specific DNA

302 packaging evolved once or multiple times, we first sought to determine more accurately which of

303 the RcGTA-like TerLs likely belong to bona fide GTAs. Shakya et al. (2017) hypothesized that

304 genomic regions with a smaller number of recognizable RcGTA gene homologs are more likely

305 to be prophages than GTAs because these regions tend to have a more virus-like GC content

306 relative to their host, evolve faster and are more often associated with viral genes. Therefore,

307 some of the TerLs classified as "RcGTA-like" might not belong to RcGTA-like elements in

308 cases when the alphaproteobacterial genomes that contain these genes lack homologs of other

309 RcGTA genes. Among the 527 RcGTA-like TerLs, we classified 391 as "large" (containing at

310 least 6 RcGTA-like genes near the terL gene) and 136 as "small" (1-5 RcGTA-like genes)

311 elements (Figure 2, Supplementary Table S3). 
Within the subtree that contains RcGTA-like TerLs (Figure 2), all but one of the TerLs

313 found in large elements form a well-supported clade (group 3 in Figure 2). The one TerL from a

314 large element that falls outside this clade is a representative of a cluster of three TerLs that are

315 found in the genomes of alphaproteobacteria Zavarzinia compransoris DSM 1231, Zavarzinia

316 sp. HR-AS and Oleomonas sp. K1W22B-8. This TerL belongs to a narrow "transition zone"

317 (group 2 in Figure 2) between the group 3 TerLs and the deepest branches of the subtree that

318 include exclusively viral and "virus-like" sequences (group 1 in Figure 2). The transition zone

319 also contains a mix of RcGTA-like TerLs from small elements and virus-like TerLs, including

320 the TerL from the intact prophage predicted in the genome of a planctomycete Zavarzinella

321 formosa DSM 19928. None of the TerLs within this transition zone come from functionally

322 characterized viruses or GTAs. Thus, the phylogeny indicates that RcGTA-like TerLs likely

323 evolved only once from a viral TerL, in an ancestor of group 3, by acquiring the capability to

324 package DNA non-specifically. The positions of the TerLs from Zavarzinia's and Oleomonas'

325 putative GTAs and the Zavarzinella prophage could be explained by horizontal gene transfer, as

326 previously documented in some instances for other RcGTA-like genes (Yang et al., 2017) and

327 discussed in detail below.

329 Viruses might mediate horizontal gene exchange of RcGTA-like genes

330 In addition to the above-discussed predicted prophage from Zavarzinella formosa DSM

331 19928, we identified two intact prophages in the genomes of the firmicute Thermoactinomyces

332 sp. DSM 45892 and the alphaproteobacterium Methylobacterium terrae 17Sr1-28, and 16 viruses

333 that encode TerLs that are phylogenetically most closely related to the RcGTA-like TerLs

334 (Tables 1 and 2). Notably, the TerLs of these three prophages are even more closely related 
335 phylogenetically to the TerLs from large elements than the 16 viruses are (Figure 2), but

336 whether they produce functional virions is unknown.

338 alphaproteobacteria and possess genes that are more closely related to GTA genes than to their

339 homologs in other viruses. For example, Dinoroseobacter phage vB_DshS-R5C contains

340 homologs of four putative tail genes of the RcGTA (genes g12-g15; Yang et al., 2017). The

341 predicted prophage in Zavarzinella formosa DSM 19928 encompasses a homolog of the adaptor

342 gene (g6) that is RcGTA-like in amino acid composition. These observations suggest an ongoing

343 exchange and recombination of RcGTA-like genes between viruses and alphaproteobacteria,

344 which likely explains the presence of virus-like TerLs within groups 2 and 3 (Figure 2). The

345 TerL phylogeny also indicates that such gene exchange might extend beyond alphaproteobacteria

346 because at least four bacterial TerLs within groups 2 and 3 come from non-alphaproteobacterial

347 genomes (OYV96073, WP_020474221, WP_110156686, and OQX66442). Because the viruses

348 with known hosts infect a wide range of bacteria that live in environments similar to those

349 occupied by GTA-containing alphaproteobacteria (Table 1), they either might have an

350 opportunity for gene exchange with viruses that infect GTA-containing bacteria or might be

351 capable of infecting GTA-containing bacteria, in addition to their currently known hosts.

Two amino acid changes distinguish GTA and viral TerLs

Although viral TerLs that are most closely related to RcGTA-like TerLs have not been

355 experimentally characterized, examination of amino acids that are conserved in RcGTA-like

356 TerLs from large elements (group 3 on Figure 2) but not in closely related viral TerLs (group 1

357 on Figure 2), or vice versa, might help pinpoint the changes that are important for the unique 
358 packaging strategy of GTAs. We did not identify any amino acids that are conserved in group 1

359 TerLs but not in group 3 TerLs, but found two amino acids (located at positions 282 and 292 in

360 the RcGTA TerL; RefSeq record WP_031321187) that are conserved in the group 3 TerLs but

361 not in the group 1 TerLs (Supplementary Table S4 and Supplementary Figure S1). In position

$362292,99 \%$ of the group 3 TerLs, but only $4 \%$ of the group 1 TerLs, contain cysteine, whereas

$36359 \%$ of the group 1 TerLs contain threonine. However, given that the threonine to cysteine

364 substitution results in a reduction of the number of carbons per side chain, selection for the

365 reduction in the energetic cost of GTA production (Kogay et al., 2020) cannot be excluded as a

366 driver for this substitution. In position $282,90 \%$ of the group 3 TerLs but no group 1 TerLs

367 contain proline, whereas $64 \%$ of the group 1 TerLs but only $6 \%$ of the group 3 TerLs contain

368 alanine. Proline contains two more carbons in its side chain than alanine, and therefore, this

369 substitution cannot be selected for energetic cost savings. In TerLs from bona fide GTAs of $R$.

370 capsulatus and Dinoroseobacter shibae, cysteine is found at position 292 in both proteins,

371 whereas in position 282 the Dinoroseobacter shibae TerL has proline and RcGTA TerL has

372 alanine.

373 Within the TerL protein structure, the two amino acids are located in the nuclease domain

374 (Rao and Feiss, 2015) and lie in close proximity at the opposite ends of a loop that extends

375 towards the translocating DNA (Supplementary Figure S2 and Figure 3B in Zhao et al. (2013)).

376 The importance of these residues with respect to the functionality of the GTA TerLs remains to

377 be elucidated. 


\section{Discussion}

RcGTA is hypothesized to package DNA via a headful mechanism because RcGTA

381 particles encapsidate random pieces of $R$. capsulatus' DNA, which would likely be facilitated by

382 a non-sequence-specific TerL (Casjens et al., 2005; Hynes et al., 2012). Previous experiments

383 support the hypothesis that RcGTA utilizes headful packaging because the packaged DNA

384 fragments have different sequences at the ends (Hynes et al., 2012). The large set of TerL

385 sequences now available allowed us to obtain phylogenetic evidence in support of this

386 hypothesis. Indeed, the RcGTA-like TerLs formed a well-supported, cohesive group within the

387 headful-packaging phage branch in our phylogeny (Figure 1).

388 Previous studies have reported that the RcGTA TerL was most closely related either to

389 the TerLs of T7-like viruses, which use a sequence-specific packaging mechanism (Casjens et

390 al., 2005), or to the TerLs of T4-like viruses, which employ the headful packaging mechanism

391 (Hynes et al., 2012; Lang and Beatty, 2000). However, we found that the RcGTA-like TerLs are

392 more similar to the TerLs of headful-packaging P22-like viruses. This discrepancy is likely due

393 to the vastly expanded set of viral sequences now available in GenBank and the more sensitive

394 search method that we used to identify viral TerL homologs.

395 In further support of the origin of RcGTA from a virus that employed a headful

396 packaging mechanism, several structural proteins of RcGTA have the highest sequence and

397 secondary structure similarity to the corresponding proteins in viruses that also utilize a headful

398 packaging strategy (Bárdy et al., 2020). Specifically, the tail tape measure protein of RcGTA is

399 homologous to the tail-needle protein of bacteriophage P22, domains of the RcGTA hub and

400 megatron proteins are homologous to their counterparts in bacteriophage T4, and the RcGTA 
401 stopper and tail terminator proteins are homologous to those from bacteriophage SPP1 (Bárdy et 402 al., 2020).

We identified TerLs from several viruses and predicted prophages that are

404 phylogenetically closer relatives of the RcGTA TerL than P22-like TerLs. These viruses and

405 predicted prophages either infect alphaproteobacteria or at least are found in the same

406 environments as GTA-containing alphaproteobacteria. Because the specific mechanisms of

407 headful packaging differ among phages (Bhattacharyya and Rao, 1993; Casjens et al., 1992 ,

408 2004), experimental characterization of packaging in viruses that are closely related to GTAs

409 could offer further insight into the origin of the GTA TerLs and their packaging mechanism.

410 The TerL phylogeny presented here supports the single origin of the RcGTA head-tail

411 cluster in alphaproteobacteria because large RcGTA-like elements grouped together. With the

412 newfound support for a single origin of RcGTA-like TerLs from a TerL of a headful-packaging

413 phage, we propose that a headful-packaging TerL in the RcGTA ancestor underwent key changes

414 that resulted in the switch from packaging the GTA genome to packaging random, small pieces

415 of the host genome (Hynes et al., 2012) with a substantially lower density of DNA in the capsid

416 (Bárdy et al., 2020). The selection for reduced energy cost of GTA protein production that

417 apparently occurred after the origin of RcGTA-like elements in alphaproteobacteria (Kogay et

418 al., 2020) makes it challenging to pinpoint amino acid changes in GTA TerLs that contribute to

419 this transition.

420 The loss of specificity for the GTA genome and the reduction in the DNA packaging

421 density rule out self-propagation of the GTA genome mediated by virions. Strikingly, the

422 RcGTA genes appear to be actively precluded from packaging, being the least frequently

423 packaged region in the alphaproteobacterial genome that is incorporated into the GTA particles 
424 (Hynes et al., 2012). The mechanism behind this exclusion is unknown, one possibility being that 425 intensive expression of the RcGTA genes interferes with their packaging (Hynes et al., 2012).

426 In addition to TerL, other GTA proteins that are involved in DNA packaging, including

427 TerS and portal, might contribute to the unique DNA packaging features of RcGTA. In

428 particular, the RcGTA gene $g 1$, which is adjacent to the terL gene $(g 2)$ in the RcGTA genome,

429 has been recently shown to encode TerS (Sherlock et al., 2019). Sherlock et al. (2019)

430 demonstrated that the RcGTA TerS binds non-specifically to DNA with low affinity due to the

431 absence of a specific DNA-binding domain and the retention of non-specific DNA binding

432 activity. A TerS protein with altered DNA-binding characteristics and a modified headful TerL

433 might together underlie the random packaging that is characteristic of RcGTA.

\section{Author Contributions}

436 E.E., O.Z., Y.I.W. and E.V.K. designed the study. E.E. collected data. E.E. and R.K. performed

437 the analyses. E.E., O.Z., Y.I.W., R.K. and E.V.K. interpreted the results. E.E. and O.Z. wrote the 438 initial draft of the manuscript. E.E., O.Z., Y.I.W., R.K. and E.V.K. revised the manuscript.

440 Acknowledgements

441 We thank Zhengshuang Hua for insightful discussions and help with using Dartmouth computing

442 facilities. This work was supported by the following awards from Dartmouth College to E.E.:

443 Sophomore Research Scholarship, James O. Freedman Presidential Scholarship, Thomas B.

444 Roos Memorial Fund Fellowship and a Kaminsky Undergraduate Research Award. Additionally, 445 this work was supported by an Intramural Research and Training Award from the National 
446 Institutes of Health to E.E., by the Simons Foundation Investigator in Mathematical Modeling of

447 Living Systems award \#327936 to O.Z., by the National Science Foundation award DEB-

4481551674 to O.Z., and by the Intramural Research Program of the U.S. National Institutes of

449 Health (National Library of Medicine) to Y.I.W. and E.V.K.

\section{Data Availability}

The following data is provided in our Supplementary Dataset available via FigShare

453 (https://doi.org/10.6084/m9.figshare.12191691): GenBank accession numbers of the 254,382

454 RcGTA TerL protein homologs that are taxonomically assigned to bacteria, archaea, or viruses

455 and likely include both ATPase and nuclease domains, GenBank accession numbers of the

456252,614 RcGTA TerL protein homologs that are represented by 11,051 TerLs in the tree in

457 Figure 1, GenBank accession numbers of the 11,051 amino acid sequences used for

458 reconstruction of the tree in Figure 1, alignment of 11,051 TerL amino acid sequences

459 (untrimmed and trimmed), phylogenetic tree of 11,051 TerLs shown in Figure 1, alignment of

460 amino acid sequences of 616 TerLs from the subtree shown in Figure 2, alignments and

461 phylogenetic trees of 342 and 346 TerLs used in IQ-TREE phylogenetic reconstructions. All

462 alignments are in FASTA format and all phylogenetic trees are in NEWICK format.

\section{References}

465 Altschul, S.F., Madden, T.L., Schäffer, A.A., Zhang, J., Zhang, Z., Miller, W., and Lipman, D.J. 466 (1997). Gapped BLAST and PSI-BLAST: a new generation of protein database search programs. 467 Nucleic Acids Res 25, 3389-3402. 
Arndt, D., Grant, J.R., Marcu, A., Sajed, T., Pon, A., Liang, Y., and Wishart, D.S. (2016). PHASTER: a better, faster version of the PHAST phage search tool. Nucleic Acids Res 44, W16-21.

471 Bárdy, P., Füzik, T., Hrebík, D., Pantůček, R., Thomas Beatty, J., and Plevka, P. (2020).

472 Structure and mechanism of DNA delivery of a gene transfer agent. Nat Commun 11, 3034.

473 Bhattacharyya, S.P., and Rao, V.B. (1993). A novel terminase activity associated with the DNA packaging protein gp17 of bacteriophage T4. Virology 196, 34-44.

475 Casjens, S.R. (2011). The DNA-packaging nanomotor of tailed bacteriophages. Nat Rev 476 Microbiol 9, 647-657.

477 Casjens, S.R., and Gilcrease, E.B. (2009). Determining DNA Packaging Strategy by Analysis of 478 the Termini of the Chromosomes in Tailed-Bacteriophage Virions. Methods Mol Biol 502, 91479111.

480 Casjens, S., Sampson, L., Randall, S., Eppler, K., Wu, H., Petri, J.B., and Schmieger, H. (1992). 481 Molecular genetic analysis of bacteriophage P22 gene 3 product, a protein involved in the 482 initiation of headful DNA packaging. J Mol Biol 227, 1086-1099.

483 Casjens, S., Winn-Stapley, D.A., Gilcrease, E.B., Morona, R., Kühlewein, C., Chua, J.E.H., 484 Manning, P.A., Inwood, W., and Clark, A.J. (2004). The chromosome of Shigella flexneri 485 bacteriophage Sf6: complete nucleotide sequence, genetic mosaicism, and DNA packaging. J 486 Mol Biol 339, 379-394.

487 Casjens, S.R., Gilcrease, E.B., Winn-Stapley, D.A., Schicklmaier, P., Schmieger, H., Pedulla, 488 M.L., Ford, M.E., Houtz, J.M., Hatfull, G.F., and Hendrix, R.W. (2005). The Generalized 489 Transducing Salmonella Bacteriophage ES18: Complete Genome Sequence and DNA Packaging 490 Strategy. J Bacteriol 187, 1091-1104.

491 Ding, H., Grüll, M.P., Mulligan, M.E., Lang, A.S., and Beatty, J.T. (2019). Induction of 492 Rhodobacter capsulatus Gene Transfer Agent Gene Expression Is a Bistable Stochastic Process 493 Repressed by an Extracellular Calcium-Binding RTX Protein Homologue. J Bacteriol 201.

494 Edgar, R.C. (2004). MUSCLE: a multiple sequence alignment method with reduced time and 495 space complexity. BMC Bioinformatics 5, 113.

496 Edgar, R.C. (2010). Search and clustering orders of magnitude faster than BLAST.

497 Bioinformatics 26, 2460-2461.

498 Ester, M., Kriegel, H.-P., Sander, J., and Xu, X. (1996). A density-based algorithm for 499 discovering clusters in large spatial databases with noise. In Proceedings of the Second 500 International Conference on Knowledge Discovery and Data Mining, (Portland, Oregon: AAAI 501 Press), pp. 226-231.

502 Fogg, P.C.M. (2019). Identification and characterization of a direct activator of a gene transfer 503 agent. Nat Commun 10, 595. 
504 Fogg, P.C.M., Westbye, A.B., and Beatty, J.T. (2012). One for all or all for one: heterogeneous 505 expression and host cell lysis are key to gene transfer agent activity in Rhodobacter capsulatus.

506 PLOS ONE 7, e43772.

507 Fokine, A., and Rossmann, M.G. (2014). Molecular architecture of tailed double-stranded DNA

508 phages. Bacteriophage 4, e28281.

509 Fu, Y., Macleod, D.M., Rivkin, R.B., Chen, F., Buchan, A., and Lang, A.S. (2010). High

510 diversity of Rhodobacterales in the subarctic North Atlantic Ocean and gene transfer agent

511 protein expression in isolated strains. Aquat Microb Ecol 59, 283-293.

512 Grull, M., Mulligan, M., and Lang, A. (2018). Small extracellular particles with big potential for

513 horizontal gene transfer: membrane vesicles and gene transfer agents. FEMS Microbiol Lett 365,

514 fny192.

515 Henikoff, S., and Henikoff, J.G. (1993). Performance evaluation of amino acid substitution 516 matrices. Proteins 17, 49-61.

517 Henikoff, S., and Henikoff, J.G. (1994). Position-based sequence weights. J Mol Biol 243, 574518578.

519 Hoang, D.T., Chernomor, O., von Haeseler, A., Minh, B.Q., and Vinh, L.S. (2018). UFBoot2:

520 Improving the Ultrafast Bootstrap Approximation. Mol Biol Evol 35, 518-522.

521 Hynes, A.P., Mercer, R.G., Watton, D.E., Buckley, C.B., and Lang, A.S. (2012). DNA

522 packaging bias and differential expression of gene transfer agent genes within a population 523 during production and release of the Rhodobacter capsulatus gene transfer agent, RcGTA. Mol

524 Microbiol 85, 314-325.

525 Hynes, A.P., Shakya, M., Mercer, R.G., Grüll, M.P., Bown, L., Davidson, F., Steffen, E.,

526 Matchem, H., Peach, M.E., Berger, T., et al. (2016). Functional and Evolutionary

527 Characterization of a Gene Transfer Agent's Multilocus “Genome.” Mol Biol Evol 33, 2530-

5282543.

529 Kalyaanamoorthy, S., Minh, B.Q., Wong, T.K.F., von Haeseler, A., and Jermiin, L.S. (2017).

530 ModelFinder: fast model selection for accurate phylogenetic estimates. Nat Methods 14, 587531589.

532 Katoh, K., and Standley, D.M. (2013). MAFFT Multiple Sequence Alignment Software Version 533 7: Improvements in Performance and Usability. Mol Biol Evol 30, 772-780.

534 Kogay, R., Neely, T.B., Birnbaum, D.P., Hankel, C.R., Shakya, M., and Zhaxybayeva, O. 535 (2019). Machine-Learning Classification Suggests That Many Alphaproteobacterial Prophages 536 May Instead Be Gene Transfer Agents. Genome Biol Evol 11, 2941-2953.

537 Kogay, R., Wolf, Y.I., Koonin, E.V., and Zhaxybayeva, O. (2020). Selection for Reducing 538 Energy Cost of Protein Production Drives the GC Content and Amino Acid Composition Bias in 539 Gene Transfer Agents. MBio 11, e01206-20. 
Lang, A.S., and Beatty, J.T. (2000). Genetic analysis of a bacterial genetic exchange element:

The gene transfer agent of Rhodobacter capsulatus. Proc Natl Acad Sci USA 97, 859-864.

542 Lang, A.S., Westbye, A.B., and Beatty, J.T. (2017). The Distribution, Evolution, and Roles of

543 Gene Transfer Agents in Prokaryotic Genetic Exchange. Annu Rev Virol 4, 87-104.

Lu, S., Wang, J., Chitsaz, F., Derbyshire, M.K., Geer, R.C., Gonzales, N.R., Gwadz, M., Hurwitz, D.I., Marchler, G.H., Song, J.S., et al. (2020). CDD/SPARCLE: the conserved domain database in 2020. Nucleic Acids Res 48, D265-D268.

547 McDaniel, L.D., Young, E., Delaney, J., Ruhnau, F., Ritchie, K.B., and Paul, J.H. (2010). High 548 Frequency of Horizontal Gene Transfer in the Oceans. Science 330, 50.

549 Merrill, B.D., Ward, A.T., Grose, J.H., and Hope, S. (2016). Software-based analysis of

550 bacteriophage genomes, physical ends, and packaging strategies. BMC Genomics 17, 679.

551 Nagao, N., Yamamoto, J., Komatsu, H., Suzuki, H., Hirose, Y., Umekage, S., Ohyama, T., and 552 Kikuchi, Y. (2015). The gene transfer agent-like particle of the marine phototrophic bacterium 553 Rhodovulum sulfidophilum. Biochem Biophys Rep 4, 369-374.

554 Nguyen, L.-T., Schmidt, H.A., von Haeseler, A., and Minh, B.Q. (2015). IQ-TREE: A Fast and 555 Effective Stochastic Algorithm for Estimating Maximum-Likelihood Phylogenies. Mol Biol Evol $55632,268-274$.

557 Philosof, A., Yutin, N., Flores-Uribe, J., Sharon, I., Koonin, E.V., and Béjà, O. (2017). Novel 558 Abundant Oceanic Viruses of Uncultured Marine Group II Euryarchaeota. Curr Biol 27, 13625591368.

Price, M.N., Dehal, P.S., and Arkin, A.P. (2010). FastTree 2 - Approximately MaximumLikelihood Trees for Large Alignments. PLOS ONE 5, e9490.

Rao, V.B., and Feiss, M. (2008). The Bacteriophage DNA Packaging Motor. Annu Rev Genet $42,647-681$.

564 Rao, V.B., and Feiss, M. (2015). Mechanisms of DNA Packaging by Large Double-Stranded 565 DNA Viruses. Annu Rev Virol 2, 351-378.

566 Schrödinger, LLC (2020). The PyMOL Molecular Graphics System, Version 2.4.

567 Shakya, M., Soucy, S.M., and Zhaxybayeva, O. (2017). Insights into origin and evolution of $\alpha$ 568 proteobacterial gene transfer agents. Virus Evol 3, vex036.

569 Sherlock, D., Leong, J.X., and Fogg, P.C.M. (2019). Identification of the First Gene Transfer 570 Agent (GTA) Small Terminase in Rhodobacter capsulatus and Its Role in GTA Production and 571 Packaging of DNA. J Virol 93, e01328-19.

572 Söding, J. (2005). Protein homology detection by HMM-HMM comparison. Bioinformatics 21, 573 951-960. 
574 Sokal, R.R., and Michener, C.D. (1958). A statistical method for evaluating systematic

575 relationships. Univ Kansas Sci Bull 38, 1409-1438.

576 Solioz, M., Yen, H.C., and Marris, B. (1975). Release and uptake of gene transfer agent by

577 Rhodopseudomonas capsulata. J Bacteriol 123, 651-657.

578 Steinegger, M., and Söding, J. (2017). MMseqs2 enables sensitive protein sequence searching for 579 the analysis of massive data sets. Nat Biotechnol 35, 1026-1028.

580 Westbye, A.B., O’Neill, Z., Schellenberg-Beaver, T., and Beatty, J.T. (2017a). The Rhodobacter 581 capsulatus gene transfer agent is induced by nutrient depletion and the RNAP omega subunit.

582 Microbiology 163, 1355-1363.

583 Westbye, A.B., Beatty, J.T., and Lang, A.S. (2017b). Guaranteeing a captive audience:

584 coordinated regulation of gene transfer agent (GTA) production and recipient capability by

585 cellular regulators. Curr Opin Microbiol 38, 122-129.

586 Whelan, S., and Goldman, N. (2001). A general empirical model of protein evolution derived 587 from multiple protein families using a maximum-likelihood approach. Mol Biol Evol 18, 691588699.

589 Wolf, Y.I., Kazlauskas, D., Iranzo, J., Lucía-Sanz, A., Kuhn, J.H., Krupovic, M., Dolja, V.V., 590 and Koonin, E.V. (2018). Origins and Evolution of the Global RNA Virome. MBio 9, e0232959118.

592 Yang, Z. (1994). Maximum likelihood phylogenetic estimation from DNA sequences with 593 variable rates over sites: Approximate methods. J Mol Evol 39, 306-314.

594 Yang, Y., Cai, L., Ma, R., Xu, Y., Tong, Y., Huang, Y., Jiao, N., and Zhang, R. (2017). A Novel 595 Roseosiphophage Isolated from the Oligotrophic South China Sea. Viruses 9, 109.

596 Yutin, N., Makarova, K.S., Gussow, A.B., Krupovic, M., Segall, A., Edwards, R.A., and Koonin, 597 E.V. (2018). Discovery of an expansive bacteriophage family that includes the most abundant 598 viruses from the human gut. Nat Microbiol 3, 38-46.

599 Zhao, H., Christensen, T.E., Kamau, Y.N., and Tang, L. (2013). Structures of the phage Sf6 large 600 terminase provide new insights into DNA translocation and cleavage. Proc Natl Acad Sci USA $601 \quad 110,8075-8080$.

602 Zimmermann, L., Stephens, A., Nam, S.-Z., Rau, D., Kübler, J., Lozajic, M., Gabler, F., Söding, 603 J., Lupas, A.N., and Alva, V. (2018). A Completely Reimplemented MPI Bioinformatics Toolkit 604 with a New HHpred Server at its Core. J Mol Biol 430, 2237-2243. 
bioRxiv preprint doi: https://doi. org/10.1101/2020 10.08 331884. this version posted October 9,2020 . The copyright holder for this preprint (which was not certified by peer review) is the author/funder, who has granted bioRxiv a license to display the preprint in perpetuity. It is made available under aCC-BY-ND 4.0 International license.

\section{Figures and Tables}

607

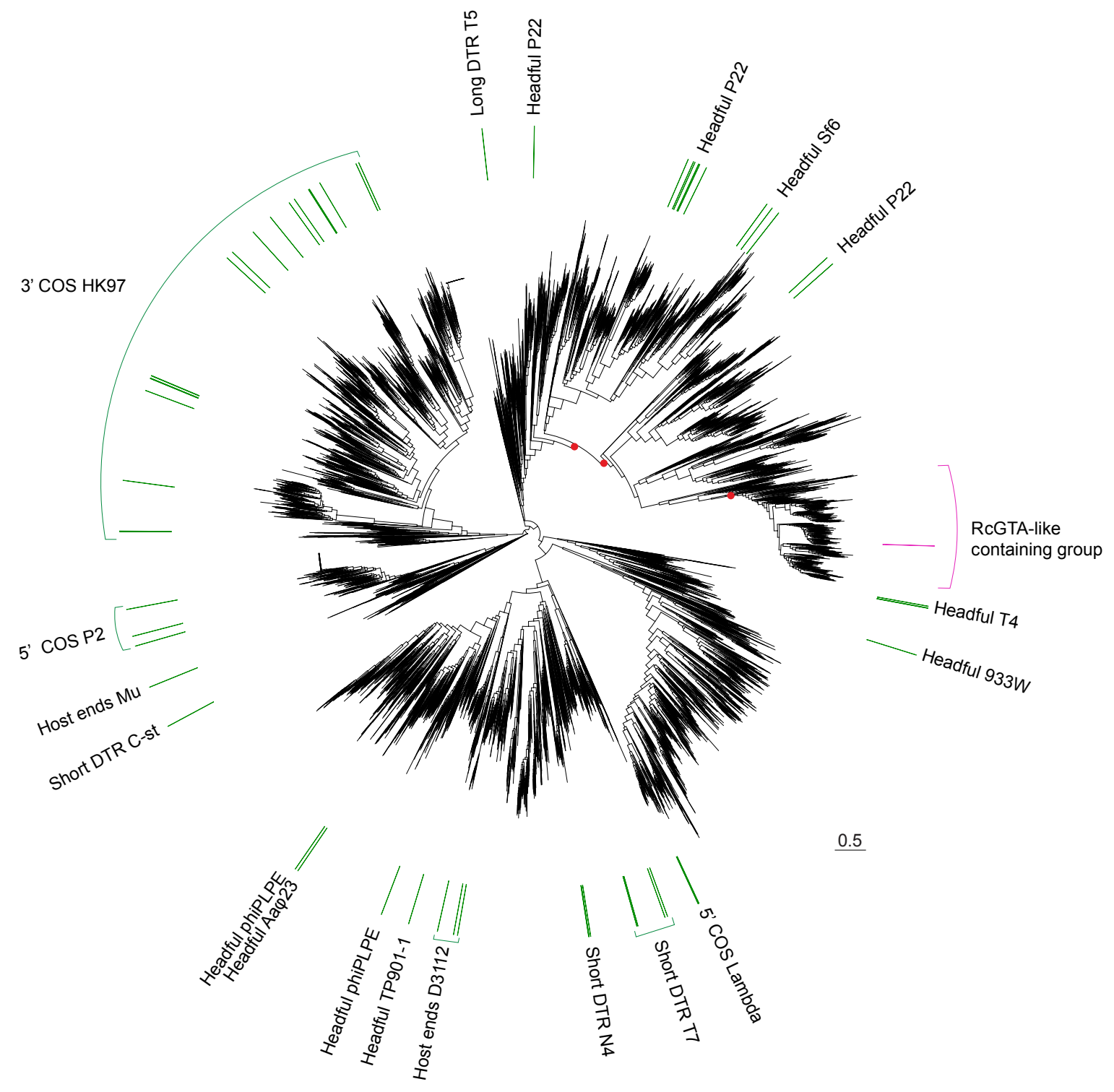

608

609

610

611

612 
613 Figure 1. Phylogeny of TerLs from 11,051 viruses, prophages and GTAs. The TerL protein

614 from RcGTA is denoted by a pink bar. The pink bracket outlines a subtree that contains RcGTA-

615 like TerLs and is shown in detail in Figure 2. The green bars denote the viruses that have

616 experimentally determined packaging strategies (Supplementary Table S2). The viruses with

617 experimentally determined packaging strategies are labeled by the packaging strategy (Headful,

618 Cohesive ends [COS], Direct Terminal Repeats [DTR], and Host Ends) followed by a prototype

619 phage from that group (e.g. P22). Support values (aLRT) of $>75 \%$ denoted by red dots are only

620 shown for a selection of branches relevant to grouping RcGTA-like TerLs within phages that

621 employ a headful DNA packaging strategy. Scale bar, amino acid substitutions per site. Tree

622 topology with all support values is available in NEWICK format in Supplementary Dataset.

623 The patterns of this phylogeny are consistent with those in the phylogenies reconstructed using a

624 more accurate maximum likelihood inference carried out using the IQ-TREE program (see

625 Supplementary Dataset); in particular, the RcGTA-like TerLs continue to form a well-

626 supported group (100\% of bootstrap samples), which forms a sister group to TerLs of phages that

627 utilize a headful packaging strategy (75\% of bootstrap samples). 
bioRxiv preprint doi: https://doi.org/10.1101/2020.10.08.331884; this version posted October 9, 2020. The copyright holder for this preprint (which was not certified by peer review) is the author/funder, who has granted bioRxiv a license to display the preprint in perpetuity. It is made available under aCC-BY-ND 4.0 International license.

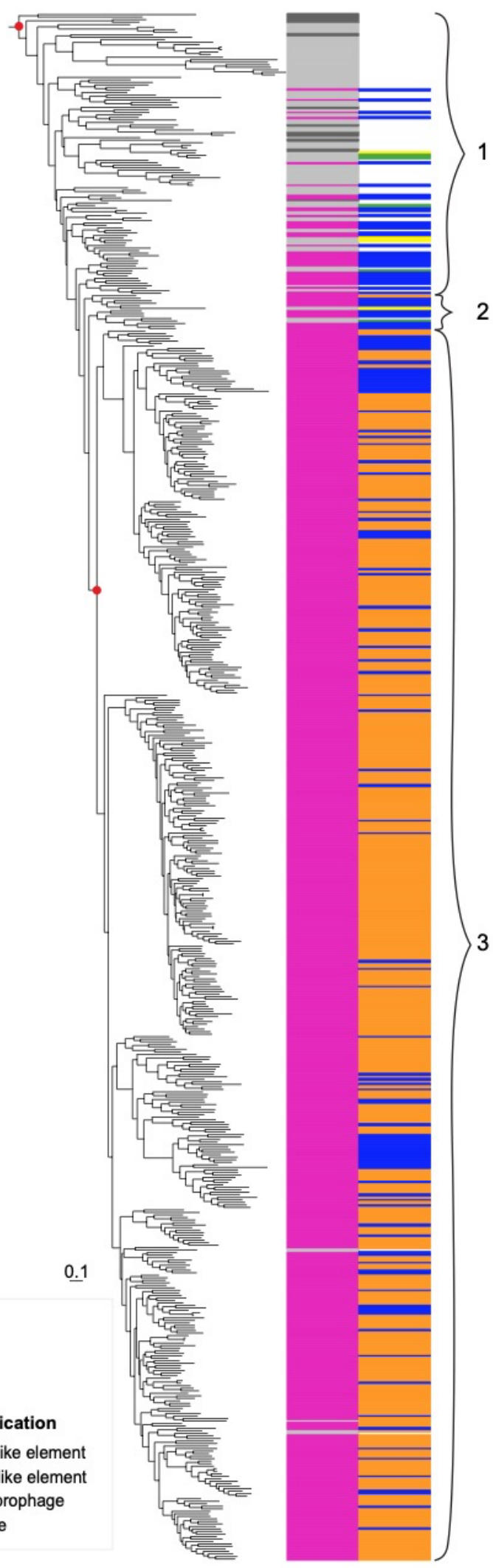


630 Figure 2. A subtree of the phylogeny shown on Figure 1 that contains RcGTA-like TerLs.

631 Bars in the first column next to the branches of the subtree indicate whether the TerLs are from

632 true viruses or, if they are found in bacterial or archaeal genomes, whether they were classified

633 by GTA-Hunter as "RcGTA-like" or "Virus-like." Bars in the second column denote whether the

634 genomic neighborhood of the terL gene contains at least 6 RcGTA-like genes ("large RcGTA-

635 like element"), between 1 and 5 RcGTA-like genes ("small RcGTA-like element"), a

636 questionable prophage or an intact prophage. TerLs without a colored bar in the second column

637 were not predicted as being in a prophage or RcGTA-like element. Group 1 includes mostly

638 virus-like TerLs as well as TerLs from predicted prophages and true viruses. Group 2 contains a

639 mixture of TerLs from a large element, small RcGTA-like elements and predicted prophages.

640 Group 3 mostly contains RcGTA-like TerLs from "true” GTAs (large RcGTA-like elements).

641 Red dots indicate two nodes that are relevant for the grouping of the entire subtree and for the

642 TerLs in group 3, and have aLRT support values of $>75 \%$. The patterns of this phylogeny are

643 consistent with those in the phylogeny reconstructed using IQ-TREE (see Supplementary

644 Dataset); in particular, the node corresponding to group 3 has $96 \%$ bootstrap support. Scale bar, 645 amino acid substitutions per site. 
Table 1. Sixteen viruses with TerLs most closely related to RcGTA-like TerLs.

\begin{tabular}{|c|c|c|c|c|c|c|c|}
\hline Virus & $\begin{array}{l}\text { TerL } \\
\text { Accession } \\
\text { Number }\end{array}$ & $\begin{array}{l}\text { On } \\
\text { tree? }\end{array}$ & Host Name & $\begin{array}{l}\text { Host Taxonomic } \\
\text { Class }\end{array}$ & Habitat & GTA? ${ }^{2}$ & Reference $^{3}$ \\
\hline Arthrobacter phage Tank & ALY10550.1 & Yes & Arthrobacter sp. ATCC 21022 & Actinobacteria & Soil & No & GenBank $^{4}$ \\
\hline Arthrobacter phage Wilde & ALY10802.1 & No & Arthrobacter sp. ATCC 21022 & Actinobacteria & Soil & No & GenBank \\
\hline Caulobacter phage Sansa & AKU43425.1 & Yes & Caulobacter crescentus CB15 & $\begin{array}{l}\text { Alpha } \\
\text { proteobacteria }\end{array}$ & Aquatic & Yes & 26450723 \\
\hline Colwellia phage 9A & AFK66668.1 & Yes & $\begin{array}{l}\text { Colwellia psychrerythraea } \\
34 \mathrm{H}\end{array}$ & $\begin{array}{l}\text { Gamma } \\
\text { proteobacteria }\end{array}$ & $\begin{array}{l}\text { Cold } \\
\text { environments }\end{array}$ & No & 23224375 \\
\hline $\begin{array}{l}\text { Dinoroseobacter phage } \\
\text { vB_DshS-R5C }\end{array}$ & ARB06077.1 & Yes & $\begin{array}{l}\text { Dinoroseobacter shibae } \\
\text { DFL12T }\end{array}$ & $\begin{array}{l}\text { Alpha } \\
\text { proteobacteria }\end{array}$ & $\begin{array}{l}\text { Ocean } \\
\text { surface }\end{array}$ & Yes & 28505134 \\
\hline Gordonia phage GMA2 & AKJ72540.1 & Yes & Gordonia malaquae A448 & Actinobacteria & $\begin{array}{l}\text { Activated } \\
\text { sludge }\end{array}$ & No & 26241321 \\
\hline $\begin{array}{l}\text { Microbacterium phage } \\
\text { Hyperion }\end{array}$ & AWN03535.1 & Yes & $\begin{array}{l}\text { Microbacterium foliorum } \\
\text { NRRL B- } 24224\end{array}$ & Actinobacteria & Soil & No & GenBank \\
\hline $\begin{array}{l}\text { Microbacterium phage } \\
\text { OneinaGillian }\end{array}$ & AYB70129.1 & Yes & $\begin{array}{l}\text { Microbacterium foliorum } \\
\text { NRRL B- } 24224\end{array}$ & Actinobacteria & Soil & No & GenBank \\
\hline $\begin{array}{l}\text { Microbacterium phage } \\
\text { Squash }\end{array}$ & AWN04641.1 & No & $\begin{array}{l}\text { Microbacterium foliorum } \\
\text { NRRL B- } 24224\end{array}$ & Actinobacteria & Soil & No & GenBank \\
\hline Nitrincola phage 1M3-16 & AHX01069.1 & Yes & Nitrincola sp. 1M3-16 & $\begin{array}{l}\text { Gamma } \\
\text { proteobacteria }\end{array}$ & $\begin{array}{l}\text { Hypersaline } \\
\text { alkaline lake }\end{array}$ & No & GenBank \\
\hline Salinibacter virus M1EM-1 & AUO78912.1 & No & Salinibacter ruber M1 & Bacteroidetes & Saltern & No & 29099492 \\
\hline Salinibacter virus M8CR30-2 & AUO79033.1 & Yes & Salinibacter ruber M8 & Bacteroidetes & Saltern & No & 29099492 \\
\hline Salinibacter virus M8CR30-4 & AUO79074.1 & No & Salinibacter ruber M8 & Bacteroidetes & Saltern & No & 29099492 \\
\hline Streptomyces phage mu1/6 & ABD94197.1 & Yes & Streptomyces aureofaciens & Actinobacteria & Soil & No & 18062183 \\
\hline $\begin{array}{l}\text { environmental Halophage } \\
\text { eHP-25 }\end{array}$ & AFH22435.1 & Yes & $\begin{array}{l}\text { Unknown, hypothesized to be } \\
\text { Nanohaloarchaea }\end{array}$ & Unknown & Saltern & No & 22479446 \\
\hline $\begin{array}{l}\text { uncultured Mediterranean } \\
\text { phage uvDeep-CGR2- } \\
\text { KM21-C338 }\end{array}$ & ANS03529.1 & Yes & Unknown & Unknown & Deep ocean & No & 27460793 \\
\hline
\end{tabular}


Table 2. Three predicted intact prophages with TerLs closely related to RcGTA-like TerLs.

\begin{tabular}{|c|c|c|c|c|c|}
\hline Host Name & $\begin{array}{l}\text { TerL Accession } \\
\text { Number }\end{array}$ & $\begin{array}{l}\text { Predicted } \\
\text { prophage } \\
\text { coordinates in the } \\
\text { host's genome }\end{array}$ & Host Taxonomic Class & Host Habitat & Reference $^{1}$ \\
\hline Zavarzinella formosa DSM 19928 & WP_020474221.1 & $\begin{array}{l}\text { NZ_JH636446.1: } \\
39426-61037\end{array}$ & Planctomycetia & Wetlands & 22740668 \\
\hline Methylobacterium terrae 17Sr1-28 & WP_109959484.1 & $\begin{array}{l}\text { NZ_CP029553.1: } \\
2863294-2900746\end{array}$ & Alphaproteobacteria & Soil & 31463788 \\
\hline Thermoactinomyces sp. DSM 45892 & SDY22851.1 & $\begin{array}{l}\text { FNPL01000003.1: } \\
4278-49984\end{array}$ & Bacilli & Not Provided & $\mathrm{GenBank}^{2}$ \\
\hline
\end{tabular}

PubMed ID of the paper that discusses the isolation and/or genome of the host organism

${ }^{2}$ Direct submission to GenBank 
1 Supplementary Tables (in a separate file in Excel format)

2

Supplementary Table S1: List of the 20 profiles used as queries for PSI-BLAST searches to find TerL homologs.

Supplementary Table S2: List of 73 viral TerLs of experimentally determined packaging mechanism that were identified in our dataset of 252,614 TerL homologs.

Supplementary Table S3: GTA-Hunter classifications of 604 bacterial and archaeal predictions of prophage regions surrounding the virus-like TerLs. subtree shown in Figure 2. 


\section{Supplementary Figures}

19

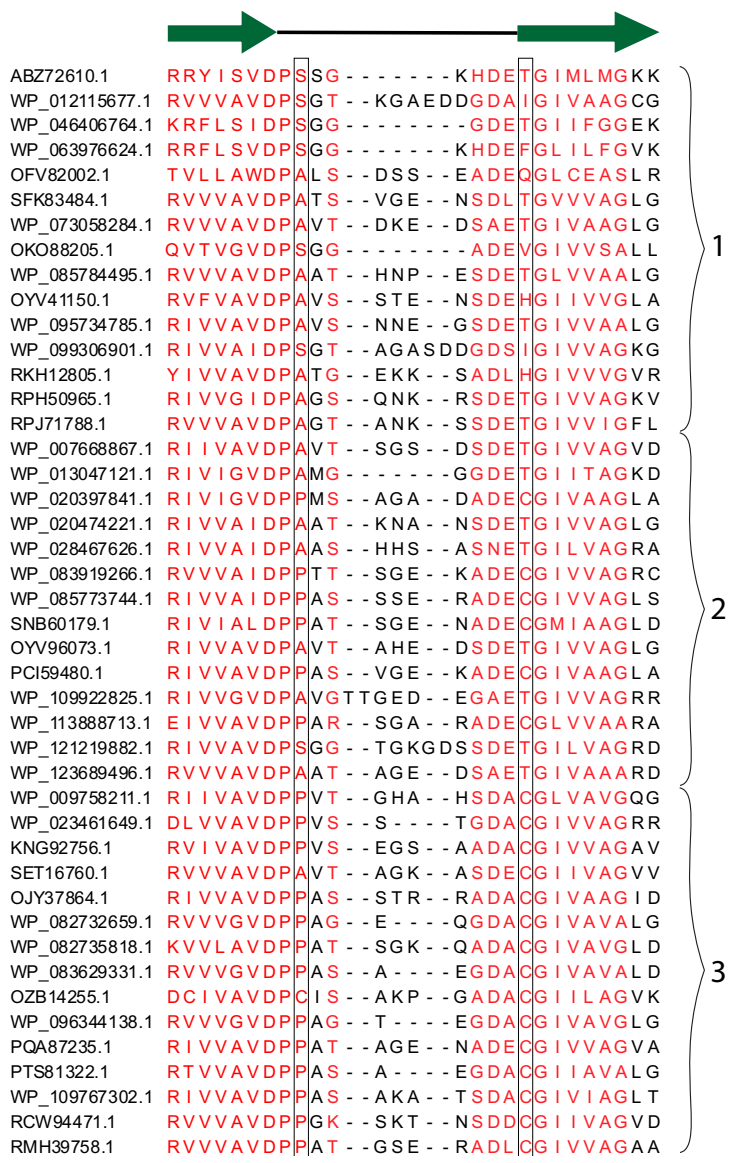

RVVVAVDPPGK - - SKT - - NSDDCGIIVAGVD

\section{Supplementary Figure S1. Multiple sequence alignment of the TerL region with two}

23 conserved sites that differentiate GTA and viral TerLs. The shown selection of representative

24 sequences is designated by their RefSeq or GenBank identifiers. The selected sequences

25 representing the three groups from Figure 2 (marked by curly braces). The secondary structure

26 of the region is shown above the alignment, where green arrows designate beta strands and the

27 black line indicates a random coil. Alignment sites colored in red contain at least 2 bits of

28 information. The two conserved differentiating sites are outlined by rectangles. The amino acids

29 found in these sites in the full dataset are provided in Supplementary Table S4. The depicted

30 region of the protein is highlighted on the TerL protein structure shown in Supplementary

31 Figure S2). 


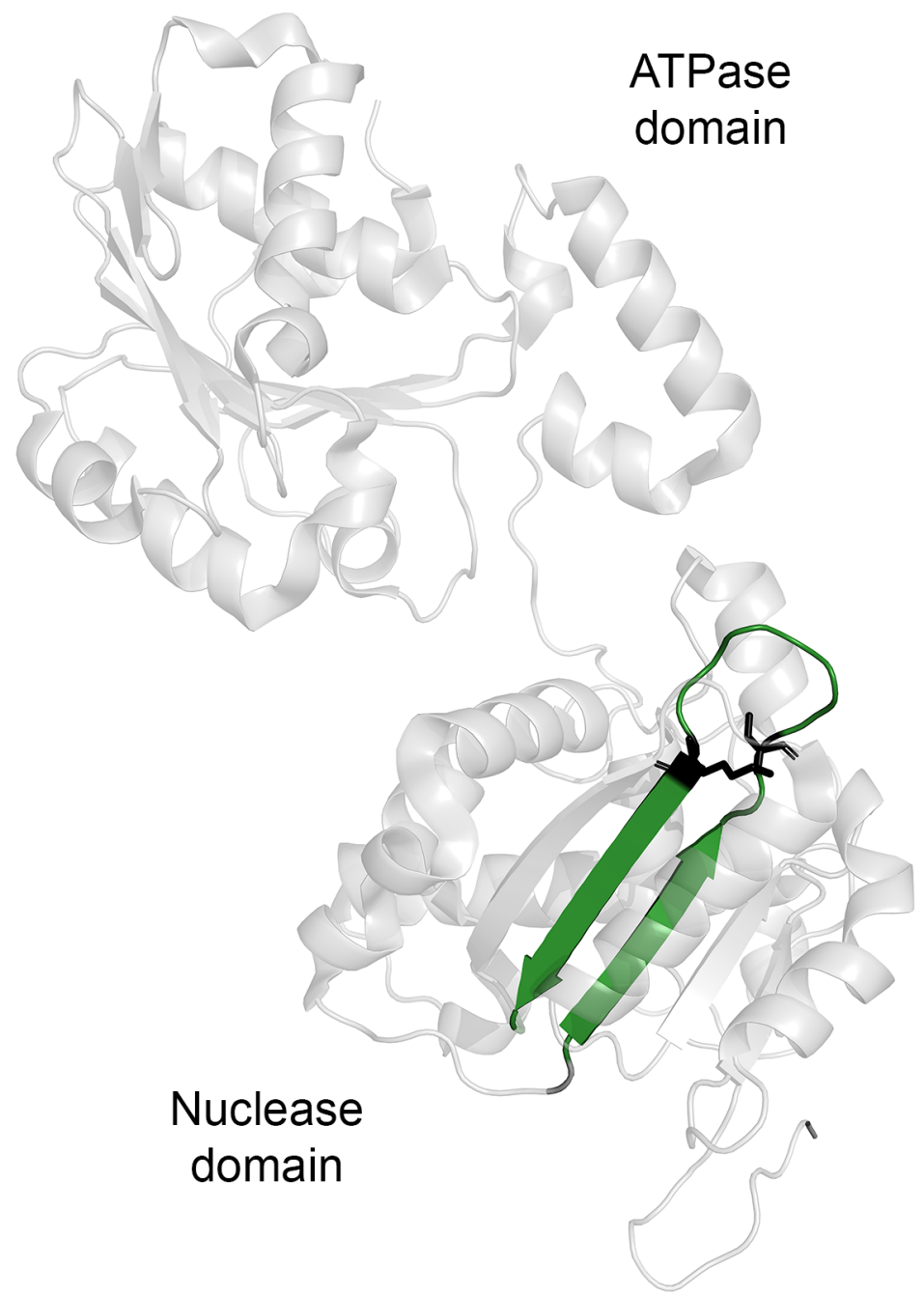

34 Supplementary Figure S2. Locations of the two conserved differentiating sites on the

35 protein structure of the TerL from Shigella phage Sf6 (PDB ID 4IDH) (Zhao et al., 2013).

36 The side chains of the two amino acids corresponding to the differentiating sites (S266 and

37 K273) are shown in black. The two beta strands and a random coil region shown in

38 Supplementary Figure S1 are colored in green. The rest of the structure is shown at reduced

39 opacity for presentation purposes. 